\title{
Defense mechanisms and quality of life of medical students according to graduation phase
}

\author{
Gisely Barddal Medeiros Borges, ${ }^{1}$ Ingrid Eidt, ${ }^{1}$ Louise Nassif Zilli, ${ }^{2}$ Ana Maria Maykot Prates Michels, ${ }^{2,3}$ \\ Alexandre Paim Diaz $^{4}$ (D)
}

\begin{abstract}
Objectives: To compare health-related quality of life (QoL) of medical students in initial and final phases of the program, and to evaluate the association between ego defense mechanisms and specific healthrelated QoL domains within each group.

Methods: This was an observational, cross-sectional study. Quality of life was assessed according to the World Health Organization Quality of Life instrument - Abbreviated Version (WHOQOL-Bref); anxiety and depression symptoms were evaluated using the Hospital Anxiety and Depression Scale (HADS); defense mechanisms were assessed using the Defense Style Questionnaire (DSQ-40).

Results: A total of 139 medical students were evaluated. Students in the initial semesters of the program (1st and 3rd) presented more depressive symptoms and worse quality of life in the psychological domain of WHOQOL-Bref when compared to those in the final semesters (8th and 12th). In a later analysis, conducted to identify the variables associated with the psychological domain of the WHOQOL-Bref for each group, both depressive symptoms and defense mechanisms were independently associated with the outcome for medical students in the beginning and in the end of the graduation program.

Conclusions: Students in the initial phases of medical school may need more specific attention from educational managers. Understanding the role of ego defense mechanisms in the quality of life of medical students may help identify effective psychopedagogical interventions for this population. In addition, the results reinforce the impact of depressive symptoms on quality of life, an association already well evidenced in the literature.
\end{abstract}

Keywords: Medical academics, quality of life, defense mechanisms, depressive symptoms.

\section{Introduction}

Quality of life (QoL) is an important outcome in health. It is defined as the "individuals' perception of their position in life in the context of the culture and value systems in which they live and in relation to their goals, expectations, standards and concerns." 1 QoL incorporates, in a complex way, the subject's physical and psychological state, their level of independence, social relations, personal beliefs and their relations with the environment. ${ }^{1,2}$ Among the several factors associated with QoL, depression is one of the most frequently found in the literature. ${ }^{3}$

Anxiety symptoms, depression and suicidal ideation are not uncommon in medical students and resident physicians. A systematic review on the prevalence of depressive symptoms and suicidal ideation in medical students observed that the prevalence of depressive symptoms in this population was higher than that reported for the general population. ${ }^{4}$ Medical school is described as a stressor that can negatively affect the academic performance, health, and psychological

\footnotetext{
${ }^{1}$ Faculdade de Medicina, Universidade Federal de Santa Catarina (UFSC), Florianópolis, SC, Brazil. ${ }^{2}$ Programa de Residência Médica em Psiquiatria, Instituto de Psiquiatria de Santa Catarina, São José, SC, Brazil. ${ }^{3}$ Departamento de Clínica Médica, UFSC, Florianópolis, SC, Brazil. ${ }^{4}$ Núcleo de Psiquiatria, UFSC, Florianópolis, SC, Brazil.

Submitted Mar 09 2019, accepted for publication Jun 272019.

Suggested citation: Borges GBM, Eidt I, Zilli LN, Michels AM, Diaz AP. Defense mechanisms and quality of life of medical students according to graduation phase. Trends Psychiatry Psychother. 2020;42(1):74-81. http://dx.doi.org/10.1590/2237-6089-2019-0022
} 
well-being of these students. ${ }^{5}$ The highly competitive selection before even entering the medical school and later the competitive university environment, not to mention the intense hours and the conviviality with people in situation of illness, can all influence the QoL of the students and medical professionals. In addition to the evaluation of QoL in the different phases of the medical training program, in order to identify phases of greater vulnerability, it is indispensable to study which variables would be associated with the students' QoL this could help identify interventions that could have an impact on their future professional performance. ${ }^{6}$

Most Brazilian medical students are in their adolescence, a period of learning and social development, when they are susceptible to peer influence and the sense of self is in development. ${ }^{7}$ The cognitive development of adolescents includes the development of more advanced reasoning skills, the ability to think abstractly and the capacity of more accurately perceiving their own feelings and how others perceive them. ${ }^{8}$ Psychosocial and emotional development is characterized by increased autonomy, the establishment of identity and better selfregulation of emotions. ${ }^{8}$ In addition, as they mature, stronger regulatory control is achieved, allowing delay in gratification and improved long-term planning. ${ }^{7}$

The Diagnostic and Statistical Manual of Mental Disorders (DSM-5) defines a defense mechanism as "mechanisms that mediate the individual's reaction to emotional conflicts and external stressors. Some defense mechanisms are almost invariably maladaptive. Others may be maladaptive or adaptive, depending on their severity, inflexibility and the context in which they occur." 9 This is a concept derived from psychoanalysis and represents unconscious resources used by the ego to reduce the conflict between the id and the superego. ${ }^{10}$

In a prospective study, George Vaillant accompanied 30 physically healthy men selected from a sample of 268 male university students with the goal of ranking ego defense mechanisms. From this study, which made it possible to map the defense styles of the participants, Vaillant classified the defenses as "immature," "neurotic," and "mature." For the author, the ego's primitive mechanisms evolve into more mature mechanisms. In addition, individuals with mature defenses would exhibit optimal adaptation to life, while those with immature defenses, only a regular adaptation. ${ }^{11}$

In the research literature, we observed that only a small number of studies evaluated the association between defense mechanisms and QoL, and few have adjusted the findings for psychiatric symptoms - a relevant confounder in QoL research. In a crosssectional study, Vojvodić et al. found that military personnel that use mature defense mechanisms have a better perception of QoL.12 Talepasand \& Mahfar evaluated the relationship between defense mechanisms and QoL among women with breast cancer in a correlational study. The authors found and inverse association between displacement and regression defense mechanisms and physical, cognitive and role aspects of QoL. ${ }^{13}$ Only one study has investigated this association in medical students. ${ }^{14}$

Thus, the objectives of this study were to compare the medical students' QoL in different phases of the medical program, and to identify the sociodemographic, clinical and defense mechanisms associated with QoL in a Brazilian sample of medical students. Considering that the impact of the imminent beginning of professional life and the greater responsibility with patients grows in importance towards the end of the program, our hypothesis was that students attending the final semesters would present worse QoL when compared to students in the initial semesters.

\section{Methods}

\section{Study design}

This was a cross-sectional, observational study.

\section{Participants}

The medical program at Universidade Federal de Santa Catarina (UFSC) is structured in 12 semesters, with activities being held in the community since the early semesters. The two first semesters are dedicated to basic theorical disciplines; from the 3rd semester onwards, focus is on the study of health conditions. The 8th semester is the last one predominantly theorical before the internship (9th to 12th semester). The use of active learning methodologies, in which students are more involved in the teaching process, despite being increasingly stimulated in the pedagogical process, is not mandatory.

Our sample comprised students attending the 1st, $3 \mathrm{rd}, 8$ th and 12 th semesters of the medical program at UFSC, Florianópolis, state of Santa Catarina, Brazil. These semesters were chosen after discussion among the researchers about which would be critical moments during medical school: 1st semester - beginning of the program; 3rd - beginning of the student's contact with clinical examination of patients; 8th - last theoretical period of the program before the internship; and 12th - last period before graduation. Students were contacted and invited to participate in the study, and received explanations about the study objectives and characteristics. Those who agreed to participate signed an informed consent form. In the second half 
of 2015, 196 students were enrolled in the 1st, 3rd, 8 th and 12th semesters of the program (52, 50, 44 and 50, respectively). Inclusion criteria were students enrolled in the 1st, 3rd, 8th and 12th semesters of the medical program in the second half of 2015 (period of data collection). The only exclusion criterion was being younger than 18 years old.

\section{Research instruments}

This study consisted of a self-applied protocol, which included a sociodemographic questionnaire, The World Health Organization Quality of Life instrument - Abbreviated Version (WHOQOL-Bref), ${ }^{15}$ the Hospital Anxiety and Depression Scale (HADS), ${ }^{16}$ and the Defense Style Questionnaire (DSQ-40) for the evaluation of defense mechanisms. ${ }^{17}$ Data on religious practice, sports and leisure activities were investigated using yes/no questions, as was information on alcohol use.

World Health Organization Quality of Life instrument Abbreviated Version (WHOQOL-Bref)

The instrument chosen to assess QoL was the WHOQOL-Bref, prepared by the World Health Organization Quality of Life Group. The WHOQOL-Bref is composed of 26 questions, two of which relate to general QoL and health, and the other 24 questions form four specific domains: psychological, physical, social relations and environment. Higher scores on the WHOQOL-Bref or its domains correspond to better QoL. This instrument has been validated for application in the Brazilian population. ${ }^{15}$ The WHOQOL-Bref is one of the most widely used research tools for assessing QoL in mental health. It accesses the individuals' perception within their cultural context and value systems, taking into account their goals and concerns.

\section{Hospital Anxiety and Depression Scale (HADS)}

The HADS scale was developed to assess anxiety and depression symptoms in patients hospitalized for non-psychiatric illnesses and it was subsequently validated for outpatients and non-diagnosed patients. HADS is a self-administered scale that can also be applied by an interviewer and is valid for measuring symptoms of anxiety and depression in individuals with non-psychiatric and psychiatric disorders. This instrument comprises 14 questions, of which seven investigate anxiety (HADS-A) and the other seven investigate depression (HADS-D). ${ }^{16}$ This instrument has been validated for use in the Brazilian population. ${ }^{18}$

\section{Defense Style Questionnaire (DSQ-40)}

Ego defense mechanisms correspond to a psychoanalytic concept and have served as indicative of the typical way an individual handles their conflicts. The DSQ-40 is a scale developed to identify how people perceive the derivatives of these defense mechanisms; to do so, it relies on questions that assess their behavior against specific situations. The DSQ-40 is composed of 40 questions, which are related to the 20 defense mechanisms described in the Diagnostic and Statistical Manual of Mental Disorders, 3rd Edition Revised (DSM-III-R). Each defense is evaluated by two questions. Four defenses are related to the mature factor (sublimation, humor, anticipation and suppression), 12 to the immature factor (projection, passive aggression, actuation, isolation, devaluation, "autistic fantasy," negation, dislocation, dissociation, cleavage, rationalization and somatization), and four to the neurotic factor (nullification, pseudo-altruism, idealization and reactive formation). ${ }^{19}$ This instrument has been validated in Portuguese. ${ }^{17}$

\section{Data analysis}

Continuous variables were described as mean and standard deviation or median and interquartile range, depending on the distribution of the data, analyzed according to the Kolmogorov-Smirnov test. The groups (represented by initial and final phases of the medical program) were compared according to QoL scores, as well as sociodemographic and clinical variables. For categorical variables, the chi-square test or Fisher's exact test were used; parametric continuous data were analyzed using the Student $t$ test. Continuous non-parametric data were analyzed using the Mann-Whitney test.

Simple linear regression analysis was used to evaluate which variables were associated with QoL domain scores for each group separately. Associations whose $p$-value resulted $<0.20$ were included in a multiple linear regression model. After these last analyses, $\mathrm{p}$-values $<0.05$ were considered significant.

\section{Ethical aspects}

The research project was approved by the research ethics committee of UFSC. All participants signed an informed consent form prior to data collection.

\section{Results}

Among the 196 students enrolled in the 1st, 3rd, 8th and 12 th periods of the medical program in the second semester of 2015,139 (71.3\%) agreed to participate in this study.

In the second half of 2015, 52, 50, 44 and 50 students were enrolled in the 1st, 3rd, 8th and 12th semesters of the program, respectively. Of these, 
$41(78.8 \%), 43(86 \%), 33(75 \%)$ and $22(44 \%)$, respectively, were evaluated. We believe that the lower rates of evaluation in the 8 th and 12 th semesters are due to an increased number of extra-class activities towards the end of the medical program. The protocol was presented to students in one opportunity at the end of theorical classes taught by authors GBMB and IE; fewer students were reached among those with more in-hospital activities. Unfortunately, we do not have information about how many participants have refused to answer the study protocol.

All evaluations were conducted in the second half of 2015 . The majority of the participants were female $(53.2 \%)$, with a mean age \pm standard deviation (SD) of $21.1 \pm 2.6,22 \pm 2.8,24.7 \pm 3$, and $25.1 \pm 2$ years old for the 1st, 3rd, 8th and 12 th semester, respectively. Less than $30 \%$ came from the metropolitan region were the medical school is located; a minority, $26.7 \%$, lived alone. Of the students evaluated, 59\% reported having a habit of practicing sports weekly, and most of the students, $74.1 \%$, reported consuming alcoholic beverages weekly.

Students attending the 1st and 3rd semesters were grouped and referred to as "initial phases," whereas students attending the 8 th and 12 th semesters were grouped and referred to as "final phases." The comparison between these two groups showed that students in the initial phases presented significantly more depressive symptoms - median of 5 points on HADS-D (interquartile range $[\mathrm{IQR}]=3-7$ ) - when compared to students in the final phases - median HADS-D score of $3(\mathrm{IQR}=2-6)(p=0.01)$. In relation to defense mechanisms, students in the initial phases also had significantly higher neurotic $(p=0.004)$ and immature ( $p=0.006)$ scores; in the assessment of QoL, students in the initial phases presented worse QoL in the psychological domain $(p=0.008)$ (Table 1 ).

Linear regression analysis was applied to identify which variables would be associated with psychological QoL scores for each group separately. It was found that, for the initial phases, depressive symptoms (coefficient B $-2.82,95 \%$ confidence interval $[95 \% \mathrm{CI}]-3.67$ to -1.97 ) and the immature factor (coefficient B $-12.82,95 \% \mathrm{CI}$ -19.30 to -5.11) were independently associated with poorer psychological QoL after multiple linear regression analysis (Tables 2 and 3). For the final phases, in addition to depressive symptoms (coefficient B -3.04, $95 \%$ CI -3.96 to -2.11 ), the neurotic factor (coefficient B -6.92, 95\%CI -12.19 to -1.65) was associated with worse psychological QoL (Tables 4 and 5).

Table 1 - Sociodemographic characteristics, psychiatric and psychological symptoms and quality of life according to medical graduation phase (initial vs. final)

\begin{tabular}{|c|c|c|c|}
\hline & $\begin{array}{c}\text { Initial phases } \\
\text { (1st and 3rd semesters) } \\
n=84\end{array}$ & $\begin{array}{c}\text { Final phases } \\
\text { (8th and 12th semesters) } \\
n=55\end{array}$ & $\mathbf{p}$ \\
\hline \multicolumn{4}{|l|}{ Sociodemographic characteristics } \\
\hline \multicolumn{4}{|l|}{ Sex } \\
\hline Male & $37(44)$ & $28(50.9)$ & 0.42 \\
\hline Female & $47(56)$ & $27(49.1)$ & \\
\hline Age in years, median (IQR) & $21(19-23)$ & $24(24-26)$ & $<0.001^{*+}$ \\
\hline \multicolumn{4}{|l|}{ Stable relationship } \\
\hline Yes & $27(32.1)$ & $26(47.3)$ & 0.07 \\
\hline No & $57(67.9)$ & $29(52.7)$ & \\
\hline \multicolumn{4}{|l|}{ Dwelling $^{\ddagger}$} \\
\hline Metropolitan region & $23(29.9)$ & $16(29.6)$ & 0.97 \\
\hline Other city & $54(70.1)$ & $38(70.4)$ & \\
\hline \multicolumn{4}{|l|}{ Living alone } \\
\hline Yes & $23(27.4)$ & $14(25.5)$ & 0.80 \\
\hline No & $61(72.6)$ & $41(74.5)$ & \\
\hline \multicolumn{4}{|l|}{ Religion practice } \\
\hline Yes & $27(32.1)$ & $13(23.6)$ & 0.27 \\
\hline No & 57 (67.9) & $42(76.4)$ & \\
\hline
\end{tabular}


Table 1 (cont.)

\begin{tabular}{|c|c|c|c|}
\hline & $\begin{array}{c}\text { Initial phases } \\
\text { (1st and 3rd semesters) } \\
n=84\end{array}$ & $\begin{array}{c}\text { Final phases } \\
\text { (8th and 12th semesters) } \\
n=55\end{array}$ & $\mathbf{p}$ \\
\hline \multicolumn{4}{|l|}{ Sport activity weekly } \\
\hline Yes & $47(56)$ & $35(63.6)$ & 0.36 \\
\hline No & $37(44)$ & $20(36.4)$ & \\
\hline \multicolumn{4}{|l|}{ Leisure activity weekly§ } \\
\hline Yes & $44(52.4)$ & $29(54.7)$ & 0.79 \\
\hline No & $40(47.6)$ & $24(45.3)$ & \\
\hline \multicolumn{4}{|l|}{ Alcohol use } \\
\hline Yes & $60(71.4)$ & $43(78.2)$ & 0.37 \\
\hline No & $24(28.6)$ & $12(21.8)$ & \\
\hline \multicolumn{4}{|c|}{ Psychiatric symptoms, mean (SD) } \\
\hline HADS-A & $8.3(4.0)$ & $7.3(4.0)$ & 0.15 \\
\hline HADS-D, median (IQR) & $5(3-7)$ & $3(2-6)$ & $0.01 * \|$ \\
\hline \multicolumn{4}{|c|}{ Psychological symptoms, mean (SD) } \\
\hline Mature factor & $2.7(0.5)$ & $2.6(0.5)$ & 0.23 \\
\hline Neurotic factor & $2.4(0.5)$ & $2.1(0.7)$ & 0.0049 \\
\hline Immature factor & $1.9(0.4)$ & $1.7(0.5)$ & $0.006^{\natural}$ \\
\hline \multicolumn{4}{|l|}{ Quality of life, mean (SD) } \\
\hline Physical domain & $61.6(15.3)$ & $65.9(16.3)$ & 0.12 \\
\hline Psychological domain & $56.9(16.9)$ & $64.4(14.6)$ & $0.008^{9}$ \\
\hline Social domain & $63.5(18.6)$ & $68.0(20.3)$ & 0.18 \\
\hline Environmental domain & $63.5(16.0)$ & $67.4(13.4)$ & 0.13 \\
\hline
\end{tabular}

Data presented as $\mathrm{n}(\%)$, unless otherwise specified.

HADS = Hospital Anxiety and Depression Scale; IQR = interquartile range; SD = standard deviation.

* Mann-Whitney test; ${ }^{\dagger} \mathrm{p}<0.001 ;{ }^{\ddagger} 8$ missing; ${ }^{\S} 2$ missing; $" \mathrm{p}<0.05 ;$ " $\mathrm{p}<0.01$.

Table 2 - Simple linear regression analysis between independent variables and quality of life psychological domain scores in the initial phases (1st and 3rd semesters) of medical school

\begin{tabular}{lccccc}
\hline Variable & B coefficient & $\mathbf{9 5 \% C I}$ & $\mathbf{R}$ & $\mathbf{R}^{\mathbf{2}}$ & $\mathbf{P}$ \\
\hline Age & -1.36 & -2.70 to -0.02 & 0.22 & 0.05 & $0.047^{*}$ \\
HADS-D & -3.38 & -4.26 to -2.50 & 0.64 & 0.41 & $<0.001^{+}$ \\
Neurotic factor & -7.35 & -14.30 to -0.40 & 0.23 & 0.05 & $0.04^{*}$ \\
Immature factor & -20.47 & -28.20 to -12.74 & 0.50 & 0.25 & $<0.001^{+}$ \\
\hline
\end{tabular}

$95 \% \mathrm{CI}=95 \%$ confidence interval; HADS-D = Hospital Anxiety and Depression Scale - Depression subscale.

$* \mathrm{p}<0.05 ;{ }^{+} \mathrm{p}<0.001$.

Table 3 - Multiple linear regression analysis to evaluate the independent associations with quality of life psychological domain scores in the initial phases (1st and 3rd semesters) of medical school

\begin{tabular}{|c|c|c|c|c|}
\hline Variable & B & $95 \% \mathrm{CI}$ & $\mathbf{p}$ & $\mathbf{R}^{2}$ \\
\hline & & & & 0.56 \\
\hline Age & -1.13 & -2.10 to -0.17 & $0.02 *$ & \\
\hline HADS-D & -2.82 & -3.67 to -1.97 & $<0.001^{+}$ & \\
\hline Neurotic factor & -2.98 & -8.36 to 2.40 & 0.27 & \\
\hline Immature factor & -12.82 & -19.30 to -5.11 & $0.001^{\ddagger}$ & \\
\hline
\end{tabular}

$95 \% \mathrm{CI}=95 \%$ confidence interval; HADS-D = Hospital Anxiety and Depression Scale - Depression subscale.

$* \mathrm{p}<0.05 ;{ }^{\dagger} \mathrm{p}<0.001 ;{ }^{\ddagger} \mathrm{p}<0.01$.

78 - Trends Psychiatry Psychother. 2020;42(1) 
Table 4 - Simple linear regression analysis between independent variables and quality of life psychological domain scores in the final phases (8th and 12th semester) of medical school

\begin{tabular}{lccccc}
\hline Variable & B coefficient & $\mathbf{9 5 \%} \mathbf{C I}$ & $\mathbf{R}$ & $\mathbf{R}^{\mathbf{2}}$ & $\mathbf{P}$ \\
\hline Age & 0.41 & -1.13 to 1.94 & 0.07 & 0.005 & 0.59 \\
HADS-D & -3.52 & -4.42 to -2.62 & 0.73 & 0.54 & $<0.001^{*}$ \\
Neurotic factor & -12.06 & -17.52 to -6.61 & 0.54 & 0.29 & $<0.001^{*}$ \\
Immature factor & -13.00 & -21.21 to -4.79 & 0.41 & 0.17 & $0.003^{+}$ \\
\hline
\end{tabular}

$95 \% \mathrm{CI}=95 \%$ confidence interval; HADS-D = Hospital Anxiety and Depression Scale - Depression subscale.

$* \mathrm{p}<0.001 ;{ }^{+} \mathrm{p}<0.01$

Table 5 - Multiple linear regression analysis to evaluate the independent associations with quality of life psychological domain scores in the final phases (8th and 12th semesters) of medical school

\begin{tabular}{lccc}
\hline Variable & B coefficient & $\mathbf{9 5 \% C I}$ & $\mathbf{p}$ \\
\hline \\
Age & & & $\mathbf{R}^{\mathbf{2}}$ \\
HADS-D & 0.11 & -0.88 to 1.11 & 0.82 \\
Neurotic factor & -3.04 & -3.96 to -2.11 & $<0.001^{*}$ \\
Immature factor & -6.92 & -12.19 to -1.65 & $0.01^{+}$ \\
\hline
\end{tabular}

$95 \% \mathrm{CI}=95 \%$ confidence interval; HADS-D $=$ Hospital Anxiety and Depression Scale - Depression subscale.

$* \mathrm{p}<0.001 ;{ }^{+} \mathrm{p}<0.05$.

The standardized residuals from both multiple linear regression analyses followed a normal distribution, according to the Kolmogorov-Smirnov normality test ( $p$ $=0.98$ and $p=0.85$ for the 1 st $/ 3$ rd and the 8th/12th semester regression models, respectively).

\section{Discussion}

In this study, medical students in the initial phases (1st and 3rd phases) of the medical program, when compared to those in the final phases (8th and 12th phases), presented significantly more depressive symptoms, worse QoL in the psychological domain and higher scores on the neurotic and immature factors of the DSQ-40. When analyzing the variables associated with the QoL psychological domain for each group separately, depressive symptoms and defense mechanisms were associated with worse QoL in the psychological domain for both groups. However, whereas for the initial phases the immature factor had an independent association with a poorer QoL in this domain, for the final phases the neurotic factor of the DSQ-40 was the variable associated with the same outcome after the multiple linear regression.

In our study, the mean score of depressive symptoms, according to HADS-D, was $5.3 \pm 3.2$ and 4.1 \pm 3.0 for students in the initial and final phases, respectively. In the study of Vasconcelos et al., which aimed to assess the prevalence of anxiety and depression symptoms in medical students, the mean
HADS-D score was $4.4 \pm 3.1,{ }^{20}$ similar to the one found in the present investigation. De Paula et al. found that the prevalence of depressive symptoms decreased as the student progressed during the period of medical school. ${ }^{21}$ Conversely, Nava et al. observed that the prevalence of depression was significantly higher among undergraduate students in the last two years of medical school than among first-year students. ${ }^{22}$ Rotenstein et al., in a systematic review of the prevalence of depressive symptoms in medical students, observed its increase by $13.5 \%$ along the course of the graduation program. ${ }^{4}$

Taking into account the information obtained by the WHOQOL-Bref questionnaire (QoL) and by the DSQ-40 (ego defense mechanisms), we observed a negative association between psychological domain scores and the immature factor for students in the initial phases, suggesting a worse quality of psychological life associated with increased immaturity. For students in the final phases, the results were similar, however for the neurotic factor of DSQ-40. The association of the psychological domain with different types of defense mechanisms in the initial and final phases of medical school reinforces the idea that ego defense mechanisms, which can influence psychological well-being, may exert different influences along the course of medical graduation. In a study conducted by physicians in the municipality of Botucatu, state of São Paulo, Brazil, with the objective of evaluating factors associated with QoL, Miranda et al. found a positive association between the psychological domain and the mature factor of DSQ-40, 
i.e., better psychological QoL was associated with higher scores on the mature factor. ${ }^{23}$ Thus, it is possible that the defenses predominantly required at the beginning, at the end of graduation and in the medical practice are distinct, transitioning between the immature factor at the beginning of graduation, through the neurotic factor at the end of graduation, and finally the mature factor when the profession is at more advanced stage of consolidation, which could be a consequence of both age and professional experience.

We observed that the students in the initial phases of the program presented lower scores in the psychological domain of the QoL when compared to those in the final phases, reflecting a worse psychological QoL of students in the 1st and 3rd periods when compared to students in the 8th and 12th ones. These findings may be related to the students' mental exhaustion resulting from the quest to ensure a place in medical school, the prospect of starting the desired program, the transition from school life to university life, and the adaptation to this new reality, both geographically and socially, since many students come from other cities and states and are distant from their family members, who are an important support network.

In a study investigating the mental health of students starting medical school in Germany, Wege et al. inferred that both the vulnerability due to the transition period into adulthood and the highly competitive environment influenced by stress could explain the poorer mental health of newcomers to medical school. ${ }^{24}$ However, contrary to our results, Alves et al. ${ }^{25}$ and Chazan et al. ${ }^{26}$ found a greater impairment of the psychological QoL in medical students in the final phases of the program.

In order to evaluate the relationship between curricular structure and well-being in medical students in the period of preclinical activity (1st and 2 nd year), Reed et al. measured the structure of the curriculum through the hours dedicated to didactic experiments, clinics and exams. The results showed that students whose curricular structure presented a greater percentage of hours with clinical activities reported less wear and stress and a lower tendency to drop out of the program. ${ }^{27}$ In other words, the absence of contact with medical practice in the early phases of graduation may also be a factor that contributes to increased stress and, consequently, a worsening in psychological QoL.

This study has several limitations that should be addressed. First, the 12th semester is probably an atypical period of medical school, since several - if not all - medical students are also preparing themselves for the medical residency exam, which could increase their stress. In addition, the 8th semester may be more similar in scope to the first half of medical school than the second one. In this sense, perhaps a comparison between the first four semesters vs. the four last ones could be more appropriate for the aims stated in this study. Second, clinical information about alcohol use lacks validity, as we have not used a validated tool for this evaluation. Third, it is not possible to discard a type II error, as we have not calculated the minimum sample size necessary for finding a specific effect size established a priori.

\section{Conclusions}

Different defense mechanisms seem to be related to psychological QoL at different moments of the medical graduation program, which may reflect not only the age and experience acquired during training, but also aspects related to the transition phase (high school to college) and curricular structure (greater volume of theoretical than practical activities). Thus, this study draws attention to an aspect often neglected in studies related to QoL, namely, the potential role of defense mechanisms for the planning of psychopedagogical strategies. In addition, the results reinforce the impact of depressive symptoms on QoL, an association already well evidenced in the literature.

\section{Acknowledgements}

We would like to thank all participants for their time and effort invested.

\section{Disclosure}

No conflicts of interest declared concerning the publication of this article.

\section{References}

1. WHOQOL Group. The World Health Organization Quality of Life assessment (WHOQOL): position paper from the World Health Organization. Soc Sci Med. 1995;41:7.

2. Fleck MP. O instrumento de avaliação de qualidade de vida da Organização Mundial de Saúde (WHOQOL-100): características e perspectivas. Cien Saude Colet. 2000;5:6.

3. Schwab B, Daniel HS, Lutkemeyer C, Neves JA, Zilli LN, Guarnieri $\mathrm{R}$, et al. Variables associated with health-related quality of life in a Brazilian sample of patients from a tertiary outpatient clinic for depression and anxiety disorders. Trends Psychiatry Psychother. 2015;37:202-8.

4. Rotenstein LS, Ramos MA, Torre M, Segal JB, Peluso MJ, Guille $\mathrm{C}$, et al. Prevalence of depression, depressive symptoms, and suicidal ideation among medical students: a systematic review and meta-analysis. JAMA. 2016;316:2214-36. 
5. Baldassin S, Andrade AG. Anxiety traits among medical students. Arq Med ABC. 2006;31:5.

6. Gonçalves SS, Silvany Neto AM. Dimensão psicológica da qualidade de vida de estudantes de Medicina. Rev Bras Educ Med. 2013;37:11

7. Court JM. Immature brain in adolescence. J Paediatr Child Health. 2013;49:883-6.

8. Sanders RA. Adolescent psychosocial, social, and cognitive development. Pediatr Rev. 2013;34:354-8; quiz 8-9.

9. American Psychiatric Association. Diagnostic and statistical manual of mental disorders 5th edition (DSM-5). Washington: American Psychiatric Association; 2013.

10. Freud $A$. The ego and the mechanisms of defense. London: Hogarth Press and Institute of Psycho-Analysis; 1937.

11. Vaillant GE. Theoretical hierarchy of adaptive ego mechanisms: a 30-year follow-up of 30 men selected for psychological health. Arch Gen Psychiatry. 1971;24:107-18.

12. Vojvodić AR, Dedić G, Dejanović SD. Defense mechanisms and quality of life in military personnel with burnout syndrome. Vojnosanit Pregl. 2019;76:9.

13. Talepasand S, Mahfar F. Relationship between defense mechanisms and the quality of life in women with breast cancer. Int J Cancer Manag. 2018;11:8.

14. Waqas A, Rehman A, Malik A, Muhammad U, Khan S, Mahmood N. Association of ego defense mechanisms with academic performance, anxiety and depression in medical students: a mixed methods study. Cureus. 2015;7:e337.

15. Fleck MP, Louzada S, Xavier M, Chachamovich E, Vieira G, Santos $L$, et al. [Application of the Portuguese version of the abbreviated instrument of quality life WHOQOL-bref]. Rev Saude Publica. 2000;34:178-83.

16. Zigmond AS, Snaith RP. The Hospital Anxiety and Depression Scale. Acta Psychiatr Scand. 1983;67:361-70.

17. Blaya C, Kipper L, Heldt E, Isolan L, Ceitlin LH, Bond M, et al. [Brazilian-Portuguese version of the Defense Style Questionnaire (DSQ-40) for defense mechanisms measure: a preliminary study]. Braz J Psychiatry. 2004;26:255-8.
18. Botega N, Ponde M, Medeiros P, Lima M, Guerreiro C. Validação da Escala Hospitalar de Ansiedade e Depressão (HAD) em pacientes epiléticos ambulatoriais. J Bras Psiquiatr. 1998;47:285-9.

19. Andrews G, Singh M, Bond M. The Defense Style Questionnaire. J Nerv Ment Dis. 1993;181:246-56.

20. Vasconcelos TC, Dias BRT, Andrade LR, Melo GF, Barbosa L, Souza E. Prevalência de sintomas de ansiedade e depressão em estudantes de medicina. Rev Bras Educ Med. 2015;39:8.

21. de Paula JDA, Borges AMFS, Bezerra LRA, Parente HV, de Paula RC, Wajnsztejn R. Prevalence and factores associated with depression in medical students. J Hum Growth Dev. 2014;24:8.

22. Nava FR, Tafoya SA, Heinze G. Estudio comparativo sobre depresión y los factores asociados en alumnos del primer año de la Facultad de Medicina y del Internado. Salud Ment. 2013;36:5.

23. Miranda B, Louza MR. The physician's quality of life: relationship with ego defense mechanisms and object relations. Compr Psychiatry. 2015;63:22-9.

24. Wege N, Muth T, Li J, Angerer P. Mental health among currently enrolled medical students in Germany. Public Health. 2016;132:92-100.

25. Alves J, Tenório M, Anjos A, Figueroa J. Qualidade de vida em estudantes de Medicina no início e final do curso: avaliação pelo WHOQOL-Bref. Rev Bras Educ Med. 2010;34:6.

26. Chazan ACS, Campos MR, Portugal FB. Qualidade de vida em estudantes de medicina da UERJ por meio do WHOQOL-Bref: uma abordagem multivariada. Cien Saude Colet. 2015;20:9.

27. Reed DA, Shanafelt TD, Satele DW, Power DV, Eacker A, Harper W, et al. Relationship of pass/fail grading and curriculum structure with well-being among preclinical medical students: a multiinstitutional study. Acad Med. 2011;86:1367-73.

\section{Correspondence:}

Alexandre Paim Diaz

Universidade Federal de Santa Catarina, Campus Universitário

88040-900 - Florianópolis, SC - Brazil

Tel.: +55 (48) 37218293

E-mail: alexandrepaimdiaz@gmail.com 